The Critical Interpretive Synthesis: An assessment of reporting practices.

Joke Depraetere $^{1,2}$, Christophe Vandeviver ${ }^{1,2}$, Ines Keygnaert ${ }^{3}$, Tom Vander Beken ${ }^{1}$

${ }^{1}$ Institute for International Research on Criminal Policy (IRCP), Faculty of Law and Criminology, Ghent University, Ghent, Belgium.

${ }^{2}$ Research Foundation-Flanders (FWO), Brussels, Belgium.

${ }^{3}$ International Centre for Reproductive Health (ICRH), Faculty of Medicine and Health Sciences, Ghent University, Ghent Belgium.

Author for correspondence: Christophe Vandeviver

Email: Christophe.Vandeviver@UGent.be

Declaration of interest: None. 


\title{
The Critical Interpretive Synthesis: an assessment of reporting practices.
}

\begin{abstract}
The importance of the Critical Interpretive Synthesis (CIS) to systematically review quantitative and qualitative research results, and to critically develop new theory is increasingly recognized, as evidenced by the recent increase in published review articles applying CIS. This paper seeks to determine the extent to which published CIS reviews are transparent in describing their methodological decisions. We analysed 77 CIS review articles published between 2006 and 2018. Overall, our findings indicate that reporting practices of CIS key features in extant reviews are still far from optimal. We recommend that authors better document their CIS design to increase transparency of their study and suggest to develop a uniform reporting standard for CIS reviews to assist authors, journal editors, and reviewers in their evaluation of the scientific quality of CIS studies. The list of key features identified in this study could serve as an initial framework to augment reporting standards of CIS.
\end{abstract}

Key-words: Qualitative, Quantitative, Transparency, Reporting, Trustworthiness.

\section{Introduction}

A growing number of review methods for qualitative or quantitative research are available (for an overview of existing review methods, see Schick-Makaroff, MacDonald, Plummer, Burgess, \& Neander, 2016). However, few review methods allow to integrate results from quantitative and qualitative research. In response to this need, Dixon-Woods, Cavers, et al. (2006) developed the Critical Interpretive Synthesis (CIS) review method to provide synthesising arguments in the form of a coherent theoretical framework from both qualitative and quantitative research, generating new results in a way that is relevant and informing for both policy and future research (Barnett-Page \& Thomas, 2009; Dixon-Woods, Cavers, et al., 2006). While the demand for CIS initially came from health policy-makers and managers (Dixon-Woods, Cavers, et al., 2006), other social scientists now also benefit from such a synthesis.

However, theory development reviews are usually less explicit with regard to the methods they used (Templier \& Paré, 2017). This lack of transparency is problematic since transparent and uniform reporting of key methodological elements is essential for study quality, trustworthiness of the review, and the development of a cumulative body of scientific evidence. The quality of CIS reviews is therefore largely dependent on the transparent and uniform reporting of its key elements. 
In this paper, we evaluate reporting practices of CIS reviews and seek to develop a reporting standard to assist authors, referees, and journal editors in their reporting and assessment of CIS designs. We begin with a description of the importance of transparent reporting in literature reviews followed by an explanation of the CIS methodology. Next we report the outcome of the assessment of the available CIS literature. We conclude with recommendations for future research practices.

\section{The importance of transparent reporting}

Literature reviews play an important role in gathering and enhancing knowledge, developing new theories, and providing grounds for future research in various fields (Paré, Tate, Johnstone, $\&$ Kitsiou, 2016). Similar to research articles, transparency with regard to data and methods is essential to evaluate study quality and enhance reproducibility. Methodologically sound techniques should be used and reported to limit biases, errors and misinterpretations that can otherwise limit the value of the review and its use for future research (Altman \& Moher, 2013). Research articles that to do not live up to these standards are deemed unacceptable for publication by referees and journal editors. Similarly, a lack of transparency, including an unclear reporting of key methodological designs, elements, and procedures used in a literature review, limits its reproducibility and, therefore, the overall quality of the review. Reproducibility of literature reviews, however, should focus on the applied methodological design as such and not on the findings since this is highly dependent on the author's judgment (Templier \& Paré, 2017).

The recent bulk of review methods and development of new approaches and techniques causes confusion as to what methodological designs could be considered as a sound method for reviewing literature (Templier \& Paré, 2017). These misconceptions create a lack of agreement regarding key methodological aspects, quality, terminology, and characteristics of transparency of the various review genres that should be reported (Paré et al., 2016; Templier \& Paré, 2017). As a result, the need for uniform reporting standards is increasingly important.

In their assessment of reporting practices in various review types, Templier and Paré (2017) found theory development reviews to be less explicit in their description of their literature search and selection strategy, quality assessment, and data analysis compared to other review types such as meta-analysis or scoping review. Templier and Paré (2017) discuss various good practices and generate recommendations to increase the transparency in theory development reviews. First, the iterative nature of the literature search and selection in these 
type of reviews should be made clear by reporting each decision the authors make. Doing so allows the readers to judge if the applied methods and decisions are appropriate for the research question. Second, the authors should report whether or not a form of quality assessment is carried out when searching and selecting articles for further analysis. Third, theory development reviews should be explicit in describing the used methods for data extraction by clearly describing the coding procedure used to identify themes and concepts as well as how the literature was categorized.

While these recommendations set the base for reporting standards, they remain broad and lack a detailed description of key methodological elements per specific review type. In this paper, we focus on one specific review genre, the CIS, which is a type of theory development review. This relatively new review method is increasingly used by various scholars across disciplines and offers methodological means to produce explanatory theories from a large and diverse set of literature (Bales \& Gee, 2012). By assessing the reporting practices of extant CIS reviews we aim to develop a reporting standard that is grounded in research practice and could aid future scholars using the CIS method when describing their methodological decisions to increase the trustworthiness and overall quality of their literature review.

\section{The Critical Interpretive Synthesis}

The CIS provides a systematic, empirical method for combining both qualitative and quantitative forms of research (Bales \& Gee, 2012). The technique builds on existing review methods, including an adaptation of the meta-ethnography, and uses techniques (i.e. method for analysis) from the grounded theory and processes (i.e. gathering of literature) from the systematic review (Bales \& Gee, 2012; Barnett-Page \& Thomas, 2009; Dixon-Woods, Cavers, et al., 2006). Systematic reviews, on the one hand, are designed to summarize the findings of the literature under well-specified and pre-defined categories and to test theories that answer 'what works' questions. This approach is limited when the purpose is to generate theory. The meta-ethnography and grounded formal theory, on the other hand, have been developed to synthesize qualitative research results. CIS was developed to address these limitations and enable a synthesis of a complex body of literature from diverse types of research evidence (Dixon-Woods, Cavers, et al., 2006).

The integration of both quantitative and qualitative empirical studies, conceptual and empirical papers, reviews, and commentaries into a single review framework is one of the major advantages of CIS reviews that distinguish it from other literature review approaches (SchickMakaroff et al., 2016). According to the primary authors, combining both research methods 
may provide "insightful and illuminating ways of understanding the phenomena" (DixonWoods, Bonas, et al., 2006, p.40) and is considered to be useful and relevant for both policymakers, practice, and future research (Dixon-Woods, Cavers, et al., 2006).

The goal of CIS is to generate new theory. The different theoretical categories, generated from the available evidence, will be integrated to create an overarching theory, leading to a more profound understanding of the topic under study (Dixon-Woods, Cavers, et al., 2006; Schick-Makaroff et al., 2016). In contrast with other review methods that limit their critical orientation to the exclusion or inclusion of papers in their analyses, CIS relies on a dynamic and recursive approach. The critique forms "a key part of the synthesis, informing the sampling and selection of material and playing a key role in theory generation "(Dixon-Woods, Cavers, et al., 2006, p.6).

Another related distinctive feature is its rejection of a "stage" approach. The dynamic, iterative and interactive process of question formulation, source search and selection, and analysis distinguishes it from a systematic review. The need for flexibility is explicitly acknowledged and formal data extraction methods could be a valid strategy for data extraction, but may also be unnecessary for the purposes of a CIS and a burdensome process (DixonWoods, Cavers, et al., 2006). The constant reflexivity during the process fuels the development of emerging theoretical notions and guides the search of articles (Dixon-Woods, Cavers, et al., 2006; Schick-Makaroff et al., 2016).

Overall six general categories of activities can be identified which represent the dynamic process of the CIS:

1) Open research question: The CIS starts with the formulation of an open research question which will be refined during the execution of a CIS and will not be finalized until the end of the review.

2) Literature search: A broad searching strategy (e.g., website search, reference chaining, contacting experts) is initiated in addition to a more structured approach.

3) Literature selection: Literature is selected based on likely relevance, including purposive selection with flexible inclusion criteria (not necessarily aiming to identify and include all relevant literature). The ongoing selection of the literature should be informed by the emerging conceptual framework based on the principles of theoretical saturation.

4) Quality appraisal: Quality appraisal is based on content, likely relevance and theoretical contribution. Papers that are considered to be 'fatally flawed' may be excluded from the synthesis. To assess the quality of the paper, Dixon- Dixon-Woods, Cavers, et al. (2006) 
used five questions focussing on the aims of the research, research design, research process, amount of data and, method of analysis. The exclusion based on quality is usually deferred until the synthesis phase since papers considered to be methodologically weak may still provide relevant insights regarding the emerging theoretical framework.

5) Data extraction: The CIS demands constant reflexivity with an ongoing critical orientation to the material by placing the literature within its context. The construction of a theoretical framework starts with the analysis of the papers using similar analysis techniques as the meta-ethnography. In doing so recurring themes are identified using language from the studies themselves.

6) Formulation of a synthesising argument: Finally, the concepts are constantly compared with the data in order to identify the relationships among them. The aim is to develop a synthesising argument in the form of a coherent theoretical framework including a network of constructs and the relationships between them.

Dixon-Woods, Cavers, et al. (2006); Entwistle, Firnigl, Ryan, Francis, and Kinghorn (2012); Haddrill (2017); Schick-Makaroff et al. (2016).

\section{Method}

The aim of this review is to assess the reporting practices in current CIS reviews by evaluating how many elements of the CIS method are actually reported in the available literature and establish a reporting standards of its key elements. In doing so, we also provide an overview of CIS publications since its inception in 2006. The outcome of the review allows us to assess the quality of CIS reporting.

\section{Literature Search}

Potentially relevant sources were searched in four academic databases: Google scholar, Web of Science, Pubmed and PyschInfo from October until the beginning of December 2018. Sources were searched from 2006 onwards (i.e. year in which the CIS has been developed). Only one search term was used: 'critical interpretive synthesis'. This yielded 360 results. After removal of duplicates $(\mathrm{N}=160)$, titles were screened and abstracts were read. Flexible inclusion criteria were used to ensure that a substantial range of CIS reviews were captured. The sources were considered inappropriate if the implementation of a CIS could not be derived from the title or abstract. This lead to the exclusion of 94 articles. If one of the following terms was visible in either the title or abstract, this was considered as enough indication that a CIS was conducted: 
- Critical interpretive synthesis

- Systematic review of qualitative research

- Interpretive synthesis

- Synthesis of qualitative and quantitative research

- Critical review

\section{[INSERT FIGURE 1]}

The full-text of 106 papers were assessed for eligibility. Another 29 articles were excluded based on the following criteria:

1) No methodological or protocol papers discussing the CIS method, since these sources do not include an actual implementation of the CIS;

2) No conference proceedings or presentations, since these sources do not include a full report of the CIS; and

3) Full-text is unobtainable.

Ultimately, we analyzed 77 sources in this paper (an overview of which can be found in Appendix I), including 70 journal articles, three doctoral dissertations, three master theses, and one book chapter.

\section{Data Extraction}

A data extraction template was developed to retrieve relevant elements from the sources included for review. The year of publication, source type, research field according to the Web of Science research areas, search methods, inclusion of qualitative, quantitative or mixed method studies, applied inclusion/exclusion criteria, sampling process, type of quality appraisal, data extraction and critical analysis method, and presence of a theoretical framework were summarized in the template.

This was followed by an assessment of the reporting of the CIS. Based on the characteristics of the CIS (see above), various distinctive features were identified, named A to $\mathrm{G}$ (view table 1). The CIS reviews were separately scored based on these key features. The papers either received the score ' 1 ' (Yes; key feature is reported), '2' (No; key feature is reported, but not implemented in line with the CIS method) or ' 3 ' (Not specified; no precise information about this key feature is given). The total score of the reporting of the CIS was calculated by summing the number of key features that were reported (score ' 1 '), with scores ranging from 0 to 7.

The first author assessed the quality of reporting in each study retained in the analysis. To guarantee sufficient inter-reliability and an objective assessment of the studies, the co-authors 
conducted an independent assessment on $13 \%$ of the studies. No significant disagreements occurred (scores between co-authors differed by no more than one point on average) and discrepancies were discussed until consensus was achieved. After discussion between all authors, the operationalisation of the key features was further clarified to ensure an objective assessment of the features.

\section{[INSERT TABLE 1]}

It is important to stress that the studies are scored based on the reporting of the key features of a CIS. However, Dixon-Woods, Cavers, et al. (2006) and Entwistle et al. (2012) often state that a CIS key feature 'may be present'. Our operationalization of the key features and assessment of these features are in this sense stricter than initially intended by the authors but are preferred in light of replicability and transparency of research.

For example, the authors of the CIS method state that a broad searching strategy should be used. However, the authors did not clarify what should be considered as 'broad'. We therefore consider the presence of at least three different searching methods as a broad strategy. Since the aim is to identify as many potentially relevant studies as possible, the strategy is considered to be automatically broad if experts were consulted, since they may directly provide authors with additional relevant sources.

In addition, the authors of the CIS method emphasise that literature selection should be driven by likely relevance. However, we felt that another aspect should be added to this feature, since many CIS reviews applied specific selection criteria. Even though most selection criteira were used to give direction to the selection of the sources (and are in this sense flexible), the emphasis on the study design of the sources may be considered as contradictory to the CIS method. Therefore, selection criteria are considered as flexible when no statements about specific study digns are made.

A final note should be made with regard to the extent of transparent reporting of the methodological decisions. Even if a key feature is reported, variations are still possible in regards to the amount of detail for describing a methodological decisions. Since an objective assessment of the extent of transparency is unfeasible, we assessed wheter or not a key feature is described and performed accoring to the CIS methodology. We did not evaluate the level of detail offered by the original authors.

\section{A hierarchy of key features}


Not every key feature is, however, crucial in CIS reporting. In fact, a hierarchy of key features can be described. Based on the emphasis that is placed on the key features by previous scholars (Dixon-Woods, Cavers, et al., 2006; Entwistle et al., 2012), the following hierarchy was applied (listed from most to least important):

1) Feature F - data extraction: since the goal of a CIS is to formulate a synthesising argument, the identification of themes is required and may therefore be considered as an important element of the CIS method.

2) Feature G - synthesising argument: placed second in the hierarchy since the development of a theoretical framework is the major goal of the CIS and cannot be formulated without the idenfitication of themes (cf. feature F).

3) Feature D - literature selection: inclusion of quantitative, qualitative, and/or mixed method research is placed third given the emphasis that scholars place on this advantage.

4) Feature B - literature selection: since the CIS review aims to include as many potentially relevant studies as possible, the utilization of flexible criteria is placed fourth in the hierarchy.

5) Feature E - quality appraisal: placed fith in the hierarchy since one can also carry out a CIS without quality appraisal of the included sources.

6) Feature A - literature search: the two-staged sampling method strategy may allow the authors to detect more potentially relevant sources for the CIS review. However, a synthesising argument may also be formulated without a two-staged sampling method.

7) Feature C - literature search: placed last in the hierarchy since the broadness of the search strategy does not guarantee that more relevant sources will be found. Furthermore, a CIS review can also be conducted when no broad searching strategy is applied.

\section{Results}

\section{An Overview of CIS Publications}

Since the development of the CIS in 2006, a total of 77 studies have applied the CIS method and reviews applying the CIS method have increased from one study in 2007 to 17 studies in 2018. In particular, CIS is increasingly applied since 2015 (view figure 2). This may be due to an increased awareness of the CIS. Along with an increase of CIS studies, we noticed that methodological papers discussing the CIS method, were published starting from 2012 
$(\mathrm{N}(2012)=1, \mathrm{~N}(2014)=1, \mathrm{~N}(2016)=1, \mathrm{~N}(2017)=3) .{ }^{1}$ The publication of methodological papers may therefore lead to an increase in the recognition and thus the application of CIS.

\section{[INSERT FIGURE 2]}

Note: Life Sciences \& Biomedicine (LS\&B), Social Sciences (SS), Arts \& Humanities (A\&H)

Given the origin of the CIS method in health equity research, it is unsurprising that the majority of CIS reviews remains being published within the field of health care/policy sciences and services (view figure 3). Web of Science (WoS) research areas were used to identify fields of study. These various research areas were then grouped together as shown in figure 3. Finally the grouped research areas fall under three of the five broad WoS categories (i.e. Arts \& Humanities (A\&H), Life Science \& Biomedicine (LS\&B) and Social Sciences (SS)). Since various grouped research areas could be placed in multiple broad WoS categories, categories were combined. Overall, 51 studies were published within the category LS\&B, 21 studies within SS, four studies within the combined category LS\&B/SS and one study in the combined category including A\&H (view figure 3). In general, three out of four (73\%) studies were published in either the LS\&B category or combinations of categories that include LS\&B. The overarching category LS\&B thus clearly takes the upper hand in the implementation of CIS reviews. Nonetheless, the publication of CIS reviews in the other research areas seems to be increasing since 2013 (view figure 2). It seem that CIS is increasingly being applied outside health sciences, particularly in the social sciences.

\section{[INSERT FIGURE 3]}

\section{The Quality of the Reporting of a CIS}

\section{[INSERT FIGURE 4]}

Following the hierarchy of CIS key features, we found that the most important features ( $\mathrm{F}$ and G) are reported in approx. $90 \%$ of the studies (view figure 4). A majority of studies identified recurring themes (feature F), and a synthesizing argument (feature G), was identifiable in over $89 \%$ of studies. In addition, most studies included sources of various methodologies (feature

\footnotetext{
${ }^{1}$ These methodological papers were found during the search of CIS reviews, and were removed for further analysis based on exclusion criteria 1 (cf. figure 1).
} 
D), applied flexible selection criteria (feature B), and performed quality appraisal with emphasis on likely relevance and contribution to the theory that is being developed (feature $\mathrm{E}$ ). Nonetheless, features D and E included the highest amount of studies with the score ' 3 ' (not specified), reducing a transparent reporting of these methodological aspects. Moreover, few CIS reviews relied on a two-staged sampling process (feature A) or adopted a broad searching strategy to identify potentially relevant sources (feature C). Overall, over half $(55,8 \%)$ of the studies scored 5 or higher. Approx. one out of three (29.9\%) studies scored 6 or 7 on the reporting scale of CIS key features. Finally, ten studies reported only two CIS key features.

\section{A Tree map of CIS Key Features}

Based on the hierarchy of the key features, a tree map was made (figure 5). This tree map identifies various groups of reviews, indicated with letters A till F, where A represents the CIS studies that reported (almost) every key feature of the CIS. Eight studies in this group reported on every key CIS feature. Another 18 studies in the A group did either not apply a two-staged sampling method or did not use a broad searching strategy. However, since these features are of less importance to the ultimate goal of the CIS (constructing a synthesizing argument), these studies may be considered of good quality in regards to transparent reporting of the CIS key features.

Studies included in the B group $(\mathrm{N}=16)$ did either not apply quality appraisal according to the CIS methodology or were less transparent in their reporting of the methodology (i.e. more 'NS' scores).

The $\mathrm{C}$ group $(\mathrm{N}=2)$, includes one study that reported inclusion criteria focusing on specific methodological designs, thus leaving out potentially relevant studies, and one study that did not clearly report about the inclusion criteria nor the other lower ranked key features. However, since various methods were still included in the review, concepts were identified and a synthesizing argument was visible, the main objective of the CIS is still fulfilled.

\section{[INSERT FIGURE 5]}

Note: ' $Y$ ' (Yes) represents score 1, ' $N$ ' (No) represents score 2, and 'NS' (Not specified) represents score 3. 'ft. A till $G$ ' represent the key features of CIS.

The $\mathrm{D}$ group $(\mathrm{N}=15)$ includes non-transparent reports about the applied methodology. These studies did not clearly report whether or not various methods were included in their 
review. Studies in the E group $(\mathrm{N}=10)$ did not include various study designs in their review. Yet, combining various methods can be considered as something that differentiates a CIS from other review methods. Not doing so, may therefore be considered as a strong contra-indication for the appropriateness of the CIS method. In addition, three studies did not apply or report about the other key features (with the exception of feature F and G). As such, one may question the trustworthiness of these studies and wonder whether a different review method would have been more appropriate here.

Finally, the $\mathrm{F}$ group includes studies $(\mathrm{N}=8)$ that did neither report a synthesizing argument nor the identification of various themes. Since these features are ranked highest in the hierarchy, one may wonder about the trustworthiness and overall quality of these studies.

\section{Conclusions and Implications}

In this paper we focused on the reporting practices of the CIS methodology and provided an overview of CIS publications since its inception in 2006. Based on the results of the assessment, we found that most CIS reviews are carried out in the field of health care policy and sciences. Yet in recent years, CIS is increasingly applied in other scientific disciplines as well, particularly the social sciences. The recent availability of more methodological articles discussing CIS method might be a driver for this increase. Consequently, expanding the knowledge of CIS may be an important factor to increase the applicability of the CIS review to other scientific fields.

This paper also established that substantial inter-study variation exists in reporting standards of CIS reviews. Similar to the findings from Templier and Paré (2017) a lack of transparency was mostly visible in the description of the literature selection and quality assessment. Most studies did, however, describe their data analysis method notwithstanding variations in the amount of detail to describe their coding process. While some studies limit their reporting of their analysis method to a description of the various identified concepts and remain vague about the way in which these concepts were found, other studies provide a detailed description of the applied methodology and how the literature was categorized.

In some studies, key features of CIS were not applied according to the CIS methodology including the sampling method, literature selection, quality appraisal, and combination of various methods. Moreover, we experienced that assessing certain key features such as sampling method and quality appraisal, proves extremely difficult and is prone to bias. 
Overall, these variations indicate that the reporting and transparency regarding various methodological decisions are still far from optimal. The emphasis on the 'authorial voice' by Dixon-Woods, Cavers, et al. (2006) and the flexibility imbedded in the CIS methodology-key advantages offered by CIS over other review methods - may in fact introduce ambiguity in how the method is applied and reported in research. Bales and Gee (2012) have previously stated that the CIS has some practical limitations due to the difficulty of implementation. However, transparent reporting of the methodological elements and procedures is key for the trustworthiness and overall quality of a literature review (Templier \& Paré, 2017). Similar to the meta-ethnography (France et al., 2014), unambiguous reporting of the process and methodological decisions made during the review process is necessary to unlock the full potential of CIS.

Scholars should therefore be clearly informed about which elements need to be applied and reported per type of review method. Yet, the lack of transparency of the CIS methodology may also be rooted in journal author guidelines or the review process of the article, and not only due to CIS methodology itself or scholars failing to report key elements of their CIS review. In case of well know review methods, most notably the systematic review, journal editors and article referees are well aware of reporting standards and elements that should be reported in order to properly evaluate study methodology, improve transparency and assess overall scientific quality of the study. However, when journal editors and referees are much less familiar with the review method, specific demands on transparency are more difficult to make. In some cases, journal author guidelines leave little space for reporting study methodology, effectively limiting authors in their efforts for being transparent on their review method. In order to increase the transparency and quality of scientific journal articles, we advocate the development of reporting standards that clarify the specificities of review methods and provide authors, journal editors, and article referees with an overview of key elements that should be reported. In addition, we encourage editors in their efforts to increase the methodological transparency of the articles published in their journal, and recommend that journal guidelines reflect their efforts and allocate sufficient space to describe the methodological choices made by the authors.

The content of this paper increases the current knowledge and applicability of the CIS methodology. This may not only impact the potential number of CIS reviews in other scientific fields, but may also be relevant for various journals publishing literature reviews. An overview of the key features of the CIS increases the comprehension of this review method, allowing for more transparency when scholars report about the method and journal editors and referees 
evaluate the scientific quality of the review article. The hierarchy, set forth in our assessment of the methodological elements of CIS, may therefore be used as a standard for CIS reporting practices, aiding scholars in their future CIS review. We hope that our study may be considered as a starting point for developing similar studies and standards for methodological reporting in other review genres.

\section{Limitations}

The performed assessment in this paper only considered those studies that self-identify as CIS in the title or abstract. As such, we may have underestimated the total amount of CIS papers. However, the size of our final database allowed us to make tentative statements about the quality of CIS reviews in general.

Although all authors conducted an independent assessment of all the studies, the selection of papers was undertaken by one researcher (the first author). This may have introduced some bias and subjectivity. Using clear objectives, inclusion and exclusion criteria and a well-defined data-extraction template mitigated some of these risks.

A final limitation of this study concerns the operationalization of key features. As previously mentioned, many of the key features are described as something that 'may' be present in the review by the primary authors of the CIS. However, this paper only assessed whether a key feature was reported, was reported but not in accordance to the CIS method, or not reported at all. Some studies may therefore still be considered as a CIS in view of the primary authors, even though some features were not reported. However, the applied definition for the key features was necessary to allow for an objective assessment of the articles. We further tried to mitigate this limitation by reporting a tree map that visualized how many key features are present and from which point on a study deviates from the CIS method and diminishes in overall quality and trustworthiness.

\section{References}

Altman, D. G., \& Moher, D. (2013). Declaration of transparency for each research article. British Medical Journal Publishing Group, 347:f4796. [Editorial]. doi: https://doi.org/10.1136/bmj.f4796

Bales, S., \& Gee, C. (2012). Critical interpretive synthesis for informing collection decisions. Collection Building, 32(2), 51-56. 
Barnett-Page, E., \& Thomas, J. (2009). Methods for the synthesis of qualitative research: a critical review. BMC medical research methodology, 9(1), 59.

Dixon-Woods, M., Bonas, S., Booth, A., Jones, D. R., Miller, T., Sutton, A. J., . . Young, B. (2006). How can systematic reviews incorporate qualitative research? A critical perspective. Qualitative research, 6(1), 27-44.

Dixon-Woods, M., Cavers, D., Agarwal, S., Annandale, E., Arthur, A., Harvey, J., . . Smith, L. (2006). Conducting a critical interpretive synthesis of the literature on access to healthcare by vulnerable groups. BMC medical research methodology, 6(1), 35 .

Entwistle, V., Firnigl, D., Ryan, M., Francis, J., \& Kinghorn, P. (2012). Which experiences of health care delivery matter to service users and why? A critical interpretive synthesis and conceptual map. Journal of health services research \& policy, 17(2), 70-78.

France, E. F., Ring, N., Thomas, R., Noyes, J., Maxwell, M., \& Jepson, R. (2014). A methodological systematic review of what's wrong with meta-ethnography reporting. BMC medical research methodology, 14(1), 119.

Haddrill, R. (2017). A tale of two pregnancies: A Critical Interpretive Synthesis of women's perceptions about delayed initiation of antenatal care. Women and Birth, 31(3), 220231.

Paré, G., Tate, M., Johnstone, D., \& Kitsiou, S. (2016). Contextualizing the twin concepts of systematicity and transparency in information systems literature reviews. European Journal of Information Systems, 25(6), 493-508.

Schick-Makaroff, K., MacDonald, M., Plummer, M., Burgess, J., \& Neander, W. (2016). What synthesis methodology should I use? A review and analysis of approaches to research synthesis. AIMS public health, 3(1), 172.

Templier, M., \& Paré, G. (2017). Transparency in literature reviews: an assessment of reporting practices across review types and genres in top IS journals. European Journal of Information Systems, 27(5), 503-550. 


\section{Figures}

Figure 1: Flow chart literature selection
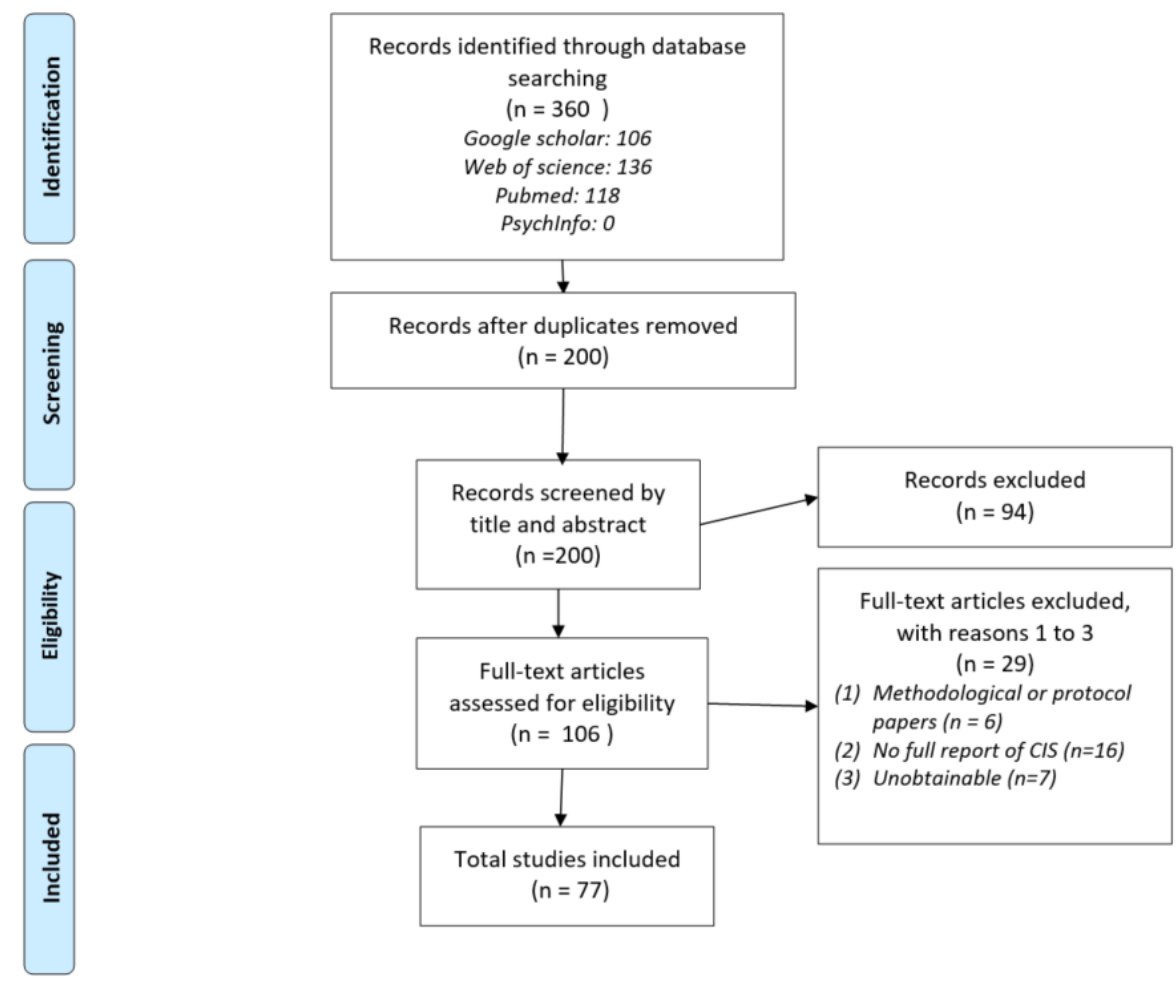

Figure 2: Fields versus year in which a CIS has been published $(\mathrm{N}=77)$

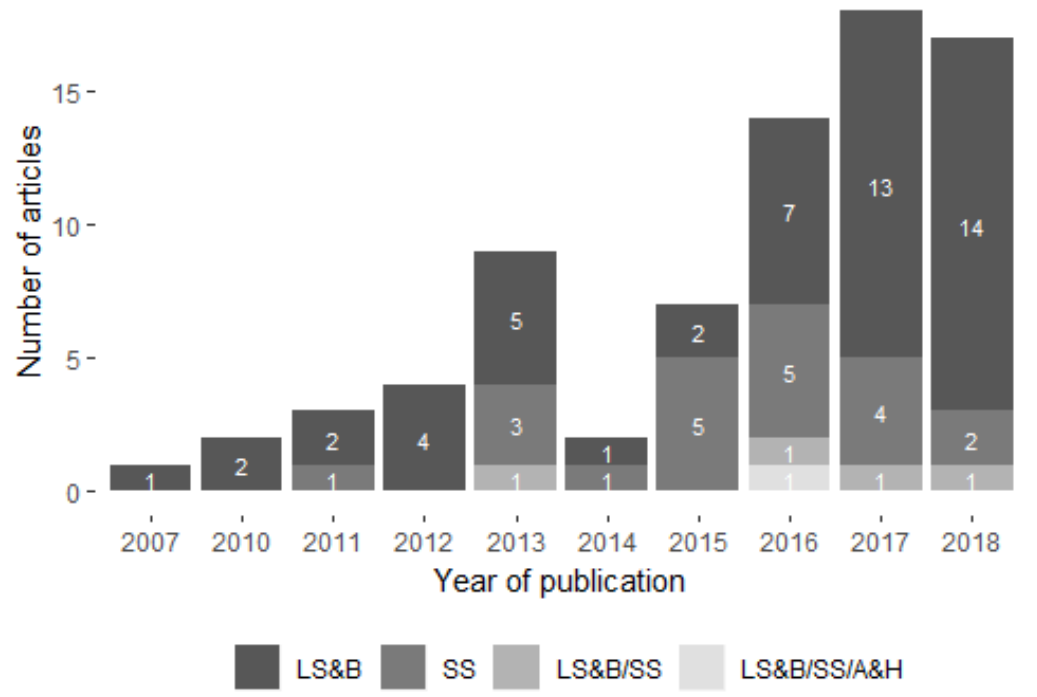

Note: Life Sciences \& Biomedicine (LS\&B), Social Sciences (SS), Arts \& Humanities (A\&H) 
Figure 3: Sankey diagram of fields in which a CIS has been published

Medical education/Nursing research: 2 = Psychiatry: 2 = Gerontology: 1 Dentistry oral medicine/ Public, Environmental \& Occupational health (PEOh): 1 Tropical medicine / (PEOh): 1 -

Rehabilitation: 4

(PEOh): 4

Health care sciences \& policy services/ (PEOh): 5

Life Science \& Biomedicine (LS\&B): 51

Medicine \& Medical Sciences: 7

Nursing: 8

CIS: 77

Health care/policy sciences \& services: 16

\section{Social Sciences (SS): 21}

Business, management, social policy \& administration: 2 = Educational research: 5

Figure 4: Scoring of key features in CIS review (Ntotal=77)

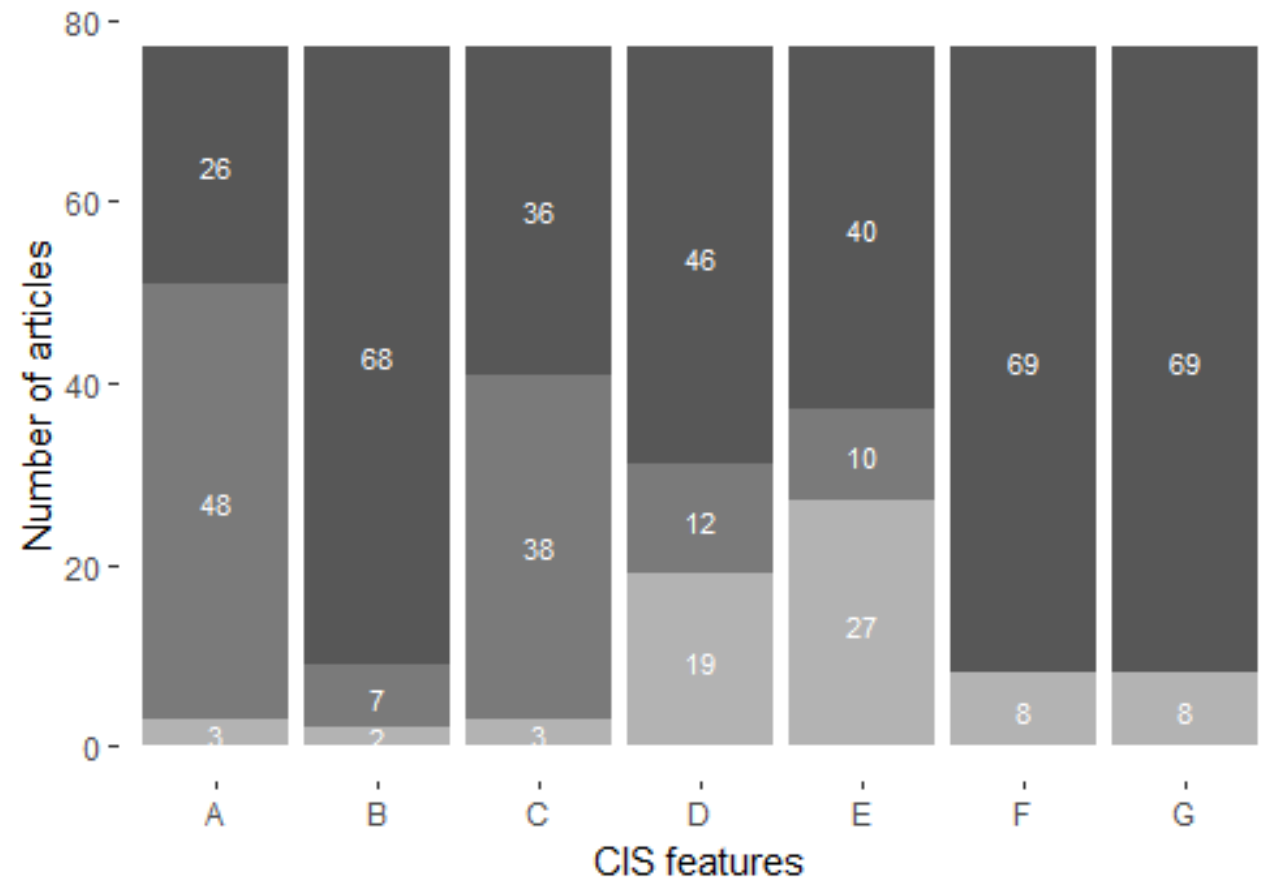

Yes No Not specified 
Figure 5: CIS tree map

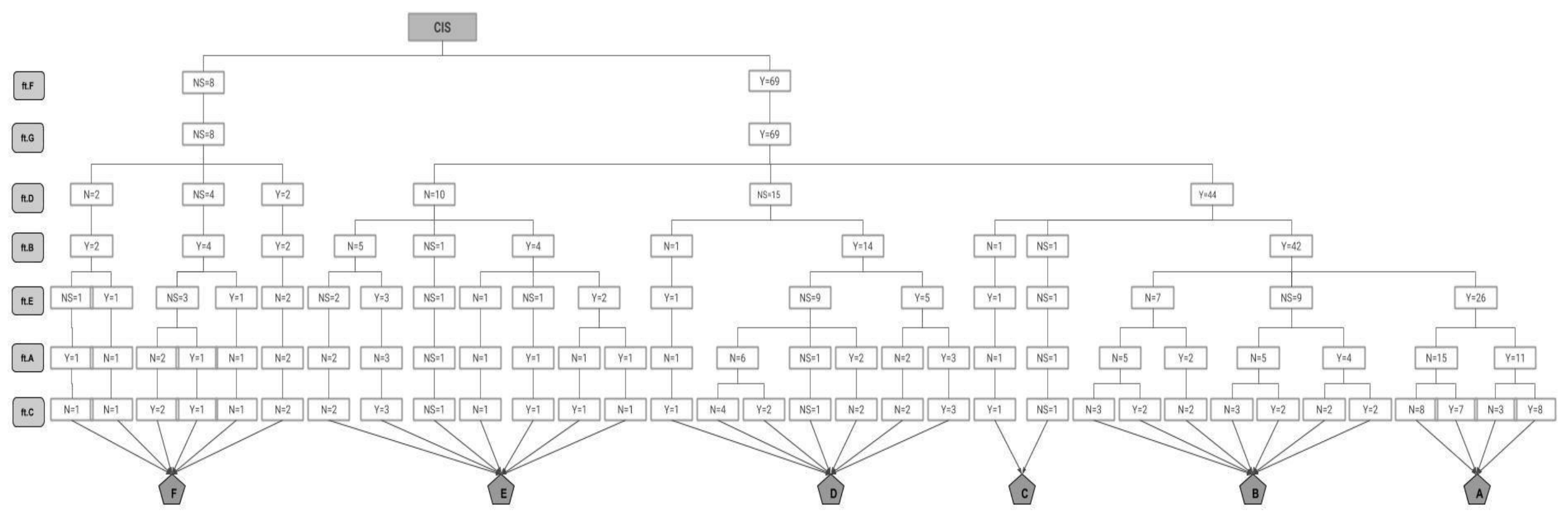

Note: 'Y'(Yes) represents score 1, 'N'(No) represents score 2, and 'NS' (Not specified) represents score 3. 'ft. A till G' represent the key features of CIS. 


\section{Tables}

Table 1: Key features of CIS

\begin{tabular}{|c|c|c|}
\hline Letter & Description of assessment criteria & Scoring \\
\hline A & $\begin{array}{l}\text { A two-staged sampling process is apparent: purposive sampling and theoretical } \\
\text { sampling based on the principles of theoretical saturation. }\end{array}$ & 1,2 or 3 \\
\hline $\mathrm{B}$ & $\begin{array}{l}\text { Flexible criteria are used. No exclusion/inclusion of studies based on used } \\
\text { methods, or no specific selection criteria are used but selection is based on } \\
\text { relevance to the research question. }\end{array}$ & 1,2 or 3 \\
\hline $\mathrm{C}$ & $\begin{array}{l}\text { A broad searching strategy is used: at least three searching methods are visible } \\
\text { (e.g., database search, reference chaining, expert consultation, website search, } \\
\text {..). If experts were consulted, the search strategy is automatically broad. }\end{array}$ & 1,2 or 3 \\
\hline $\mathrm{D}$ & $\begin{array}{l}\text { Sources with various methodologies (quantitative, qualitative or mixed methods) } \\
\text { are included. }\end{array}$ & 1,2 or 3 \\
\hline $\mathrm{E}$ & $\begin{array}{l}\text { Quality appraisal is based on likely relevance and contribution to the theory that } \\
\text { is being developed. Methodologically weak studies may be excluded, but } \\
\text { emphasis is placed on likely relevance. }\end{array}$ & 1,2 or 3 \\
\hline $\mathrm{F}$ & Recurring themes/concepts are identified. & 1or 3 \\
\hline $\mathrm{G}$ & $\begin{array}{l}\text { A synthesising argument is made by means of examining the relationship } \\
\text { between the concepts, refining the identified concepts and constructing a } \\
\text { conceptual/theoretical framework. }\end{array}$ & 1 or 3 \\
\hline
\end{tabular}




\section{APPENDIX I}

CIS publications included for assessment

Ako-Arrey, D. E., Brouwers, M. C., Lavis, J. N., Giacomini, M. K., \& Team, A.-H. (2016). Health systems guidance appraisal-a critical interpretive synthesis. Implementation Science, 11(1), 9.

Annandale, E., Harvey, J., Cavers, D., \& Dixon-Woods, M. (2007). Gender and access to healthcare in the UK: a critical interpretive synthesis of the literature. Evidence \& Policy: A Journal of Research, Debate and Practice, 3(4), 463-486.

Benjamin-Thomas, T. E., \& Rudman, D. L. (2018). A critical interpretive synthesis: Use of the occupational justice framework in research. Australian occupational therapy journal, 65(1), 3-14.

Bibb, J., Baker, F. A., \& Skewes McFerran, K. (2016). A critical interpretive synthesis of the most commonly used self-report measures in Australian mental health research. Australasian Psychiatry, 24(5), 453-458.

Cameron, J. E. (2011). Rituals surrounding the care of the dying previable baby in labour ward: a critical interpretive synthesis of the literature (Doctoral dissertation). Retrieved from: https://discovery.dundee.ac.uk/en/studentTheses/rituals-surroundingthe-care-of-the-dying-previable-baby-in-labou.

Catalano, D., Holloway, L., \& Mpofu, E. (2018). Mental Health Interventions for Parent Carers of Children with Autistic Spectrum Disorder: Practice Guidelines from a Critical Interpretive Synthesis (CIS) Systematic Review. International Journal of Environmental Research and Public Health, 15(2), 341.

Clark, M. T., Clark, R. J., Toohey, S., \& Bradbury-Jones, C. (2017). Rationales and treatment approaches underpinning the use of acupuncture and related techniques for plantar heel pain: a critical interpretive synthesis. Acupuncture in Medicine, 35(1), 9-16.

Daker-White, G., \& Rogers, A. (2013). What is the potential for social networks and support to enhance future telehealth interventions for people with a diagnosis of schizophrenia: a critical interpretive synthesis. BMC psychiatry, 13(1), 279.

De Schrijver, L., Vander Beken, T., Krahe, B., \& Keygnaert, I. (2018). Prevalence of Sexual Violence in Migrants, Applicants for International Protection, and Refugees in Europe: A Critical Interpretive Synthesis of the Evidence. International journal of environmental research and public health, 15(9), 1979.

DeFeo, N. J. (2017). How culture informs hospice music therapy: a critical interpretive synthesis (Doctoral dissertation). Retrieved from: https://dspace.sunyconnect.suny.edu/handle/1951/69212.

Degrie, L., Gastmans, C., Mahieu, L., de Casterle, B. D., \& Denier, Y. (2017). How do ethnic minority patients experience the intercultural care encounter in hospitals? A systematic review of qualitative research. Bmc Medical Ethics, 18(1), 2.

Desrosiers, J., Wilkinson, T., Abel, G., \& Pitama, S. (2016). Curricular initiatives that enhance student knowledge and perceptions of sexual and gender minority groups: a critical interpretive synthesis. Canadian medical education journal, 7(2), e121.

Du Toit, S. H. J., Shen, X., \& McGrath, M. (2018). Meaningful engagement and personcentered residential dementia care: A critical interpretive synthesis. Scandinavian journal of occupational therapy, 26(5), 343-355

Dubois, C. A., D'Amour, D., Pomey, M. P., Girard, F., \& Brault, I. (2013). Conceptualizing performance of nursing care as a prerequisite for better measurement: a systematic and interpretive review. BMC Nursing, 12(1), 7. 
Edwards, J. (2017). The extant rationale for mandated therapy during psychotherapy and counselling training: A critical interpretive synthesis. British Journal of Guidance \& Counselling, 46(5), 515-530.

Edwards, J. (2018). Counseling and Psychology Student Experiences of Personal Therapy: A Critical Interpretive Synthesis. Frontiers in psychology, 9(1732), 12.

Ellen, M. E., Wilson, M. G., Velez, M., Shach, R., Lavis, J. N., Grimshaw, J. M., \& Moat, K. A. (2018). Addressing overuse of health services in health systems: a critical interpretive synthesis. Health research policy and systems, 16(1), 48.

Endres, S., \& Weibler, J. (2017). Towards a Three-Component Model of Relational Social Constructionist Leadership: A Systematic Review and Critical Interpretive Synthesis. International Journal of Management Reviews, 19(2), 214-236.

Entwistle, V., Firnigl, D., Ryan, M., Francis, J., \& Kinghorn, P. (2012). Which experiences of health care delivery matter to service users and why? A critical interpretive synthesis and conceptual map. Journal of health services research \& policy, 17(2), 70-78.

Fairchild, R., McFerran, K. S., \& Thompson, G. (2017). A Critical Interpretive Synthesis of the Ways Children's Needs and Capacities are Represented in the Homelessness and Family Violence Literature. Children Australia, 42(1), 18-29.

Fane, J., MacDougall, C., Redmond, G., Jovanovic, J., \& Ward, P. (2016). Young children's health and wellbeing across the transition to school: a critical interpretive synthesis. Children Australia, 41(2), 126-140.

Farias, L., \& Rudman, D. L. (2016). A Critical Interpretive Synthesis of the Uptake of Critical Perspectives in Occupational Science. Journal of Occupational Science, 23(1), 33-50.

Farrelly, S., \& Lester, H. (2014). Therapeutic relationships between mental health service users with psychotic disorders and their clinicians: a critical interpretive synthesis. Health \& Social Care in the Community, 22(5), 449-460.

Flemming, K. (2010). Synthesis of quantitative and qualitative research: an example using Critical Interpretive Synthesis. Journal of Advanced Nursing, 66(1), 201-217.

Flemming, K. (2010). The use of morphine to treat cancer-related pain: a synthesis of quantitative and qualitative research. Journal of pain and symptom management, $39(1), 139-154$.

Franqueira, V. N., \& Tunnicliffe, P. (2015). To Flip or Not to Flip: A Critical Interpretive Synthesis of Flipped Teaching (pp. 57-67). In L. Uskov V., Howlett R., Jain L. (eds) Smart Education and Smart e-Learning. Smart Innovation, Systems and Technologies, vol 41. Springer, Cham: Springer.

Garrido, S., Dunne, L., Chang, E., Perz, J., Stevens, C. J., \& Haertsch, M. (2017). The Use of Music Playlists for People with Dementia: A Critical Synthesis. Journal of Alzheimer's disease, 60(3), 1129-1142.

Gysels, M. H., Evans, C., \& Higginson, I. J. (2012). Patient, caregiver, health professional and researcher views and experiences of participating in research at the end of life: a critical interpretive synthesis of the literature. BMC medical research methodology, 12(1), 123.

Haddrill, R., Jones, G. L., Anumba, D., \& Mitchell, C. (2018). A tale of two pregnancies: A Critical Interpretive Synthesis of women's perceptions about delayed initiation of antenatal care. Women Birth, 31(3), 220-231.

Harris, R. V., Pennington, A., \& Whitehead, M. (2017). Preventive dental visiting: a critical interpretive synthesis of theory explaining how inequalities arise. Community Dentistry and Oral Epidemiology, 45(2), 120-134.

Haugen, I., Sletteb $\varnothing$, T., \& Ytrehus, S. (2018). Factors affecting user participation for elderly people with dementia living at home: a critical interpretive synthesis of the literature. European Journal of Social Work, 1-13. doi: 10.1080/13691457.2018.1441133 
Heaton, J., Corden, A., \& Parker, G. (2012). 'Continuity of care': a critical interpretive synthesis of how the concept was elaborated by a national research programme. International journal of integrated care, 12, e12.

Hock, R. M., \& Mooradian, J. K. (2013). Defining coparenting for social work practice: A critical interpretive synthesis. Journal of Family Social Work, 16(4), 314-331.

Jarvis, T. (2017). Defining public health systems: A critical interpretive synthesis of how public health systems are defined and classified (Master's thesis). Retrieved from: http://hdl.handle.net/11375/22170.

Johnson, M., Tod, A. M., Brummell, S., \& Collins, K. (2015). Prognostic communication in cancer: a critical interpretive synthesis of the literature. European journal of oncology nursing, 19(5), 554-567.

Kangasniemi, M., Kallio, H., \& Pietilä, A. M. (2014). Towards environmentally responsible nursing: a critical interpretive synthesis. Journal of Advanced Nursing, 70(7), 14651478 .

Karemere, H., Kahindo, J., Ribesse, N., \& Macq, J. (2013). Governance of tertiary referral hospitals in the Democratic Republic of the Congo: a critical interpretive synthesis of the literature. Medecine et sante tropicales, 23(4), 397-402.

Kazimierczak, K. A., Skea, Z. C., Dixon-Woods, M., Entwistle, V. A., Feldman-Stewart, D., N'Dow J, M., \& MacLennan, S. J. (2013). Provision of cancer information as a "support for navigating the knowledge landscape": findings from a critical interpretive literature synthesis. European journal of oncology nursing, 17(3), 360-369.

Keliddar, I., Mosadeghrad, A. M., \& Jafari-Sirizi, M. (2017). Rationing in health systems: A critical review. Medical journal of the Islamic Republic of Iran, 31, 47.

Kelly, C. A., \& Maden, M. (2015). How do health-care professionals perceive oxygen therapy? A critical interpretive synthesis of the literature. Chronic Respiratory Disease, 12(1), 11-23.

Keygnaert, I., \& Guieu, A. (2015). What the eye does not see: a critical interpretive synthesis of European Union policies addressing sexual violence in vulnerable migrants. Reproductive health matters, 23(46), 45-55.

Lin, S., \& Melendez-Torres, G. (2017). Critical interpretive synthesis of barriers and facilitators to TB treatment in immigrant populations. Tropical Medicine \& International Health, 22(10), 1206-1222.

Mackenzie, M., Conway, E., Hastings, A., Munro, M., \& O'Donnell, C. (2013). Is 'candidacy'a useful concept for understanding journeys through public services? A critical interpretive literature synthesis. Social Policy \& Administration, 47(7), 806825.

Manthorpe, J., \& Iliffe, S. (2011). Social Work with Older People-Reducing Suicide Risk: A Critical Review of Practice and Prevention. British Journal of Social Work, 41(1), 131-147.

Markoulakis, R., \& Kirsh, B. (2013). Difficulties for university students with mental health problems: A critical interpretive synthesis. The Review of Higher Education, 37(1), 77-100.

Mathieson, A., Grande, G., \& Luker, K. (2018). Strategies, facilitators and barriers to implementation of evidence-based practice in community nursing: a systematic mixedstudies review and qualitative synthesis. Primary Health Care Research \& Development, 1-11.

McDonough, B. A. (2014). Critical Information Literacy in Practice: An Interpretive Synthesis (Doctoral dissertation). Retrieved from: https://eric.ed.gov/?id=ED545370 
McFerran, K. S. (2016). Contextualising the relationship between music, emotions and the well-being of young people: A critical interpretive synthesis. Musicae Scientiae, 20(1), 103-121.

McFerran, K. S., Garrido, S., \& Saarikallio, S. (2016). A critical interpretive synthesis of the literature linking music and adolescent mental health. Youth \& Society, 48(4), 521538.

McIntyre, L., Tougas, D., Rondeau, K., \& Mah, C. L. (2016). “In”-sights about food banks from a critical interpretive synthesis of the academic literature. Agriculture and Human Values, 33(4), 843-859.

McIsaac, J.-L., Warner, G., Lawrence, L., Urquhart, R., Price, S., Gahagan, J., . . J Jackson, L. A. (2018). The application of implementation science theories for population health: A critical interpretive synthesis. Aims Public Health, 5(1), 13.

McKibbin, G., Humphreys, C., \& Hamilton, B. (2016). Prevention-enhancing interactions: a Critical Interpretive Synthesis of the evidence about children who sexually abuse other children. Health \& social care in the community, 24(6), 657-671.

Medcalf, L., \& McFerran, K. S. (2016). A critical interpretive synthesis of music therapy case studies: Examining therapeutic boundary themes in the context of contemporary practice. British Journal of Music Therapy, 30(1), 22-35.

Moat, K. A., Lavis, J. N., \& Abelson, J. (2013). How contexts and issues influence the use of policy-relevant research syntheses: a critical interpretive synthesis. The Milbank Quarterly, 91(3), 604-648.

Morgan, H. (2016). 'Pushed'self-tracking using digital technologies for chronic health condition management: a critical interpretive synthesis. Digital Health, 2. doi: $10.1177 / 2055207616678498$

Morgan, H. M., Entwistle, V. A., Cribb, A., Christmas, S., Owens, J., Skea, Z. C., \& Watt, I. S. (2017). We need to talk about purpose: a critical interpretive synthesis of health and social care professionals' approaches to self-management support for people with long-term conditions. Health Expectations, 20(2), 243-259.

Morgan, R. L., Kelley, L., Guyatt, G. H., Johnson, A., \& Lavis, J. N. (2018). Decisionmaking frameworks and considerations for informing coverage decisions for healthcare interventions: a critical interpretive synthesis. Journal of clinical epidemiology, 94, 143-150.

Morrison, L. G., Yardley, L., Powell, J., \& Michie, S. (2012). What design features are used in effective e-health interventions? A review using techniques from critical interpretive synthesis. Telemedicine and e-Health, 18(2), 137-144.

Mothupi, M. C., Knight, L., \& Tabana, H. (2018). Measurement approaches in continuum of care for maternal health: a critical interpretive synthesis of evidence from LMICs and its implications for the South African context. BMC health services research, 18(1), 539.

Murphy, M. A., \& McFerran, K. (2017). Exploring the literature on music participation and social connectedness for young people with intellectual disability: A critical interpretive synthesis. Journal of Intellectual Disabilities, 21(4), 297-314.

Nicholas, D. B., Attridge, M., Zwaigenbaum, L., \& Clarke, M. (2015). Vocational support approaches in autism spectrum disorder: a synthesis review of the literature. Autism, $19(2), 235-245$.

Perski, O., Blandford, A., West, R., \& Michie, S. (2017). Conceptualising engagement with digital behaviour change interventions: a systematic review using principles from critical interpretive synthesis. Translational behavioral medicine, 7(2), 254-267.

Pfeffer, D., Wigginton, B., Gartner, C., \& Morphett, K. (2018). Smokers' Understandings of Addiction to Nicotine and Tobacco: A Systematic Review and Interpretive Synthesis 
of Quantitative and Qualitative Research. Nicotine and Tobacco Research, 20(9), 1038-1046.

Puurveen, G., Baumbusch, J., \& Gandhi, P. (2018). From Family Involvement to Family Inclusion in Nursing Home Settings: A Critical Interpretive Synthesis. Journal of Family Nursing, 24(1), 60-85.

Rega, M. L., Telaretti, F., Alvaro, R., \& Kangasniemi, M. (2017). Philosophical and theoretical content of the nursing discipline in academic education: A critical interpretive synthesis. Nurse Education Today, 57, 74-81.

Reidy, C., Bracher, M., Foster, C., Vassilev, I., \& Rogers, A. (2018). The process of incorporating insulin pumps into the everyday lives of people with Type 1 diabetes: A critical interpretive synthesis. Health Expectations, 21(4), 714-729.

Rudman, D. L., Egan, M. Y., McGrath, C. E., Kessler, D., Gardner, P., King, J., \& Ceci, C. (2016). Low Vision Rehabilitation, Age-Related Vision Loss, and Risk: A Critical Interpretive Synthesis. Gerontologist, 56(3), E32-E45.

Schroerlucke, D. (2015). Choke artists and clutch performers: A Critical Interpretive Synthesis (Doctoral dissertation). Available from ProQuest Dissertations Publishing. (3643162)

Shen, X. (2017). Unveiling the Concept of Person-centered Dementia Care within Occupational Therapy in Residential Aged Care Facilities: A Critical Interpretive Synthesis (Master's thesis). Retrieved from: http://hdl.handle.net/2123/16256

Talseth, A. G., \& Gilje, F. L. (2011). Nurses' responses to suicide and suicidal patients: a critical interpretive synthesis. Journal of clinical nursing, 20(11-12), 1651-1667.

Talseth, A. G., \& Gilje, F. L. (2017). Liberating burdensomeness of suicide survivorship loss: A Critical Interpretive Synthesis. Journal of clinical nursing, 26(23-24), 3843-3858.

Talseth, A. G., \& Gilje, F. L. (2018). Responses of persons at risk of suicide: A critical interpretive synthesis. Nursing Open, 5(4), 469-483.

Tetui, M., Zulu, J. M., Hurtig, A. K., Ekirapa-Kiracho, E., Kiwanuka, S. N., \& Coe, A. B. (2018). Elements for harnessing participatory action research to strengthen health managers' capacity: a critical interpretative synthesis. Health research policy and systems, 16(1), 33.

Tierney, E., McEvoy, R., O'Reilly-de Brun, M., de Brun, T., Okonkwo, E., Rooney, M., .. . MacFarlane, A. (2016). A critical analysis of the implementation of service user involvement in primary care research and health service development using normalization process theory. Health Expectations, 19(3), 501-515.

Wakefield, D., Bayly, J., Selman, L. E., Firth, A. M., Higginson, I. J., \& Murtagh, F. E. (2018). Patient empowerment, what does it mean for adults in the advanced stages of a life-limiting illness: A systematic review using critical interpretive synthesis. Palliative Medicine, 32(8), 1288-1304.

Yazdani, S., \& Akbarilakeh, M. (2017). Which Health Cares Are Related to the Family Physician? A Critical Interpretive Synthesis of Literature. Iranian Journal of Public Health, 46(5), 585.

Yazdani, S., Lakeh, M. A., Ahmady, S., Foroutan, A., \& Afshar, L. (2015). Critical interpretive synthesis of the concept of value in medical education. Research and Development in Medical Education, 4(1), 31. 